\title{
Laparoscopic Retractor
}

National Cancer Institute

\section{Source}

National Cancer Institute. Laparoscopic Retractor. NCI Thesaurus. Code C79091.

A surgical instrument designed to maneuver internal anatomic structures during laparoscopic procedures, usually to permit an unobstructed view of the area of interest. 